\title{
Ocio y usos del tiempo libre en adolescentes de 12 a 16 años en España'
}

Laura Varela Crespo"

Rita Gradaílle Pernas"

Yésica Teijeiro Bóo"
I- Estudio realizado en el marco del proyecto Tiempos escolares y tiempos de ocio: problemáticas especificas y alternativas pedagógico-sociales en la vida cotidiana del alumnado de educación secundaria obligatoria (PSI2008-04368/PSIC) que tiene su continuidad en los proyectos denominados $D e$ los tiempos educativos a los tiempos sociales: la construcción cotidiana de la condición juvenil en una sociedad de redes. Problemáticas específicas y alternativas pedagógicosociales (proyecto coordinado EDU201239080-C07-00) y Educar el ocio: realidades y perspectivas en clave intergeneracional integral e inclusiva en una sociedad de redes (EDU2015-65638-C6-1-R).

II- Universidad de Santiago de Compostela, Santiago de Compostela, España. Contactos: laura.varela@usc.es;

rita.gradaille@usc.es;

yesica.teijeiro@usc.es

\section{Resumen}

El artículo se centra en el estudio de los usos del tiempo libre y las prácticas de ocio de los adolescentes escolarizados (1216 años) en Educación Secundaria Obligatoria en España. En concreto, se presentan algunos de los resultados derivados de un proyecto de investigación enmarcado en el Plan Nacional de I+D+i (investigación científica, desarrollo e innovación tecnológica) cuyo objetivo principal era conocer e interpretar cómo inciden los tiempos escolares en los procesos de socialización de la adolescencia, así como los modos de configurar su tiempo libre. En la investigación se analizan algunos de los datos obtenidos del cuestionario diseñado ad hoc para el alumnado y aplicado a una muestra de 3306 chicos y chicas de esta franja etaria en centros educativos de las diecisiete Comunidades Autónomas que conforman el territorio español. Los análisis ponen de manifiesto el papel socializador del ocio digital, la importancia del grupo de iguales y la familia, las prácticas de ocio nocturno y la escasa participación de los adolescentes en actividades de acción social y voluntariado. Además, se señalan algunos de los desafíos que conciernen al campo pedagógico-social en la tarea de educar el ocio adolescente así como a la necesidad de una responsabilidad compartida entre los diversos agentes educativos en este proceso.

\section{Palabras clave}

Adolescencia - Educación secundaria - Tiempos de ocio - Educación del ocio. 


\section{Free time and its uses by 12- to 16-year-old adolescents in Spain'}

Laura Varela Crespo"

Rita Gradaílle Pernas"

Yésica Teijeiro Bóo"

I- Study conducted under the project Tiempos escolares y tiempos de ocio: problemáticas específicas y alternativas pedagógico-sociales en la vida cotidiana del alumnado de educación secundaria obligatoria (PSI2008-04368/PSIC -School time and free time: specific proble$m s$ and pedagogical-social alternatives in the daily life of students in compulsory secondary education), which continued in projects called De los tiempos educativos a los tiempos sociales: la construcción cotidiana de la condición juvenil en una sociedad de redes. Problemáticas específicas y alternativas pedagógicosociales (proyecto coordinado EDU201239080-C07-00 - From educational times to social times: the daily construction of the youth condition in a network society. Specific problems and social and educational alternatives) and Educar el ocio: realidades y perspectivas en clave intergeneracional integral e inclusiva en una sociedad de redes (EDU2015-65638C6-1-R - Educating free time: realities and perspectives in a comprehensive and inclusive intergenerational key in a network society).

II- Universidad de Santiago de Compostela,

Santiago de Compostela, España.

Contacts: laura.varela@usc.es;

rita.gradaille@usc.es;

yesica.teijeiro@usc.es

\section{Abstract}

This article focuses on the uses of free time and on free time practices of schooled 12- to 16-year-old adolescents attending compulsory secondary education in Spain. It presents some of the results of a research project under the National Plan of Scientific Research, Technological Development and Innovation whose main objective was to understand and interpret how school times impact on the socialization processes of adolescents, and the organization of their free time. We have analyzed some of the data obtained from a questionnaire designed ad hoc for students and applied to a sample of 3306 boys and girls of this age group in schools of the seventeen Autonomous Communities that comprise the Spanish territory. Analyses have evidenced the socializing role of digital entertainment, the importance of peer groups and family, evening leisure practices, and adolescents' low participation in social action activities and volunteer work. In addition, we indicate some of the challenges in the educational and social fields concerning the task of educating adolescent free time, as well as the need for a responsibility shared by the various educational agents involved in this process.

\section{Keywords}

Adolescence - Secondary education - Free time - Free time education. 


\section{Introducción}

Desde los años ochenta del pasado siglo, coincidiendo con los inicios del desarrollo del Estado del Bienestar en España, las problemáticas asociadas a la condición juvenil han tenido una notable presencia en la investigación de las Ciencias Sociales y Humanas (Sociología, Pedagogía, Psicología, etc.), destacando el año 1985 como fecha emblemática en la que tuvieron lugar varias circunstancias clave referidas a los jóvenes (COMAS, 2007). Entre ellas cabe destacar la celebración del Año internacional de la juventud, que actuó como un revulsivo en el desarrollo de las políticas de juventud en España y en otros países; así como la creación del Instituto de la Juventud de España (INJUVE), con la publicación continuada de informes en España -el primero en 1985 y el más reciente en el 2012-, y que han constituido un importante referente al aportar datos contrastados y bases teóricas de carácter interdisciplinar en diferentes áreas (educación, empleo, participación, asociacionismo, ocio, etc.).

En concreto, de entre los múltiples escenarios en los que se desarrolla la vida cotidiana de los adolescentes y jóvenes, sus experiencias de ocio y los usos que realizan del tiempo libre han sido -y continúan siendoobjeto de atención de investigadores, familias, educadores, etc., principalmente bajo el propósito de que los chicos y chicas aprovechen las oportunidades que les ofrecen y eviten los peligros y efectos negativos que puede conllevar las prácticas de riesgo (consumo de drogas, uso excesivo de tecnologías, etc.) asociadas a sus tiempos de libre disposición (TRILLA, 1999; SALVADOR, 2009; GRADAÍLLE; VARELA; DE VALENZUELA, 2016).

Cabe destacar que tener tiempo libre no es sinónimo de ocio, puesto que ese tiempo puede resultar tedioso y aburrido fruto de la inactividad o como consecuencia de una actividad que no nos resulta interesante ni estimulante (STEBBINS, 2012). El ocio -en sus múltiples dimensiones (lúdica, ecológica, solidaria, etc.)- se concibe como una experiencia compleja centrada en actuaciones positivas y libres que permiten a las personas hacer su existencia atractiva y tener una alta calidad de vida. Una educación del ocio debe fomentar valores, actitudes, conocimientos, competencias, habilidades, etc., con el fin de ofrecer alternativas plausibles a los apresurados ritmos de la vida moderna y a sus continuados robos de tiempo (CABALLO; CARIDE; MEIRA, 2011).

En este marco, adquiere importancia el reconocimiento del ocio como un derecho humano básico para la mejora de la calidad de vida de las personas (WLRA, 1994). Su desarrollo se facilita garantizando las condiciones básicas de vida, tales como seguridad, cobijo, comida, ingresos y educación; al tiempo que "se reivindica independientemente del trabajo, como espacio vital al que tenemos derecho por el hecho de existir, una experiencia que, encauzada adecuadamente, nos reporta salud, encuentro y desarrollo" (CUENCA, 1999, p. 24).

Como parte de esta reivindicación del derecho al ocio, el Programa de acción mundial para los jóvenes hasta el año 2000 y años subsiguientes de la Asamblea General de Naciones Unidas (NACIONES UNIDAS, 1996), reconoció las actividades recreativas como una de las diez esferas prioritarias en el desarrollo juvenil, constituyendo tales iniciativas un elemento necesario para prevenir y combatir problemas sociales (uso indebido de drogas, delincuencia, etc.) además de contribuir a la realización del potencial físico, intelectual y emocional de las personas jóvenes, siempre que no sean utilizadas como medio para adoctrinarlos o para excluir su participación en otros aspectos de la vida social.

El ocio es, por tanto, una necesidad y un derecho fundamental en los procesos de desarrollo de los adolescentes y jóvenes de nuestros días, no sólo a nivel individual sino también desde una perspectiva relacional como "ámbito privilegiado del kairós, de la subjetividad y las emociones” (LASÉN, 2000, p. 200); de ahí que -en clave educativa y socialsea necesario interpretarlo desde el disfrute que 
supone estar con otras personas por el mero hecho de compartir un tiempo y un espacio que permita sentirse mejor consigo mismo y con los demás, minimizando las injusticias que genera el modelo de sociedad dominante. En este sentido, el riesgo se sitúa en "conformarse, aceptar y por tanto, dar por buena la creencia de que el modelo de experiencia de ocio que desde el mercado se nos ofrece es, si no el único, sí el mejor camino para el desarrollo humano" (MONTEAGUDO, 2008, p. 108).

Cabe recordar que el ocio de los adolescentes y los jóvenes está influenciado no tanto por la cantidad de tiempo disponible como por los valores, vivencias y significados que atribuyen a las actividades en las que participan voluntariamente, de forma individual $\mathrm{y} / \mathrm{o}$ colectiva. Sin embargo, el gusto por la novedad y por la ruptura con la rutina, choca con la visión ampliamente extendida de la juventud como problema que incide en las múltiples dificultades que tienen los chicos y chicas para acceder al mercado laboral, en la escasa representación colectiva, así como en la imagen generalizada que los vincula a prácticas de ocio nocivas y de riesgo (consumo de drogas, abuso de nuevas tecnologías, etc.), con negativas consecuencias en su vida personal, familiar, escolar y/o social (ECHEBURÚA; LABRADOR; BECOÑA, 2009).

Frente a los enfoques reduccionistas que ignoran la heterogeneidad del colectivo juvenil, la educación del ocio ha de ser entendida como una educación que a nivel personal y comunitario aporta los saberes $\mathrm{y}$ recursos necesarios para que los chicos y chicas alcancen una vivencia más satisfactoria de su vida cotidiana; una circunstancia que requiere ser interpretada desde visiones más complejas. En este sentido, ni desde la perspectiva teórica ni desde la práctica sería aceptable

[...] limitar la educación para el ocio de los jóvenes a dotarlos de una serie de habilidades y recursos prácticos para que los apliquen en el ocio [...]. Es algo más hondo que incluye el mundo de las actitudes, sentimientos, vivencias y tomas de decisiones personales y sociales respecto a determinadas formas de actuar y de implicarse en la realidad (MERINO, 2001, p. 187).

Bajo estas coordenadas, asumir la tarea ya enunciada por Dumazedier (1964) de hacer del ocio un tiempo educativo, más allá del descanso o la recuperación de fuerzas para el trabajo, es uno de los logros de la Pedagogía del Ocio; cuya presencia se desarrolla en temáticas diversas como

[...] la educación permanente, la animación sociocultural, la función cultural y educativa de la ciudad, la planificación y control de los espacios de ocio, en tanto que elementos de solaz, de fiesta y de formación $\mathrm{y}$, en definitiva, se prolonga hacia la participación de los poderes públicos en la determinación de políticas completas y coherentes de ocio (PUIG; TRILLA, 1996, p. 43).

Sin embargo, esta visión del ocio orientada a la búsqueda de la creatividad, la autorrealización o la cooperación con los demás, no siempre se refleja en los usos del tiempo libre que realizan los adolescentes y jóvenes en su vida cotidiana.

En consecuencia, es necesario reflexionar acerca de si las actividades que los chicos y chicas desarrollan actualmente en su tiempo libre se orientan hacia una vivencia positiva de la experiencia de ocio o están más encaminadas al mero consumo y entretenimiento. En este doble proceso de desarrollo personal y social es donde la educación en y para el ocio resulta clave.

\section{Metodología}

Tal y como avanzábamos antes, en este artículo se analizan parte de los resultados de un proyecto que toma como referencia los 
tiempos educativos y los tiempos de ocio en la vida cotidiana de la población adolescente española escolarizada en Educación Secundaria Obligatoria. El estudio ha sido llevado a cabo por el Grupo de investigación en Pedagogía Social y Educación Ambiental (SEPA-interea) de la Universidad de Santiago de Compostela, con una trayectoria de más de dos décadas en el desarrollo de la línea de investigación denominada tiempos educativos y sociales. Este grupo comenzó su andadura en este ámbito en el año 1989 respondiendo al encargo institucional de la Consellería de Educación y Ordenación Universitaria de la Xunta de Galicia con el fin de realizar la evaluación de la jornada escolar de sesión única en Galicia, considerada por aquel entonces de carácter experimental. A ella siguieron numerosas investigaciones en esta temática de estudio centrada en diferentes colectivos y etapas del sistema educativo (primaria, secundaria y postobligatoria).

En España, el sistema educativo se estructura en los siguientes niveles: educación infantil, educación básica (primaria y secundaria obligatoria), educación secundaria postobligatoria (bachillerato y formación profesional de grado medio, enseñanzas profesionales de artes plásticas y diseño de grado medio y las enseñanzas deportivas de grado medio) y educación superior. En concreto, la Educación Secundaria Obligatoria, foco de este estudio, comprende cuatro cursos académicos divididos en dos ciclos que se cursan desde los 12 hasta los 16 años, aunque el alumnado tiene derecho a permanecer escolarizado en esta etapa educativa hasta los 18 años. Al finalizar dicha etapa, las alumnas y alumnos que hayan alcanzado los objetivos y competencias básicas obtienen el Título de Graduado/a en Educación Secundaria Obligatoria.

En esta investigación, se obtuvieron muestras representativas a nivel estatal proporcionales a las diferentes Comunidades Autónomas siguiendo un proceso aleatorio. En ese curso académico, el alumnado de Educación Secundaria Obligatoria era de 1.826.825; de ahí que con un nivel de confianza del 95\% y un margen de error del $\pm 3 \%$-atendiendo a los estratos: Comunidad Autónoma, curso, sexo, titularidad del centro y ubicación geográfica (rural-urbano)-, la muestra teórica mínima representativa era de 1110 alumnas y alumnos. Sin embargo, y dado que la aplicación se realizó en el grupo-aula, con una ratio de \pm 25 estudiantes, se optó porque el conjunto de la clase diese respuesta al cuestionario, evitando la selección intencionada y minimizando la mortandad de la muestra. De ahí que, el número de cuestionarios obtenidos fuese de 3306, superando la muestra teórica inicial y reduciendo el margen de error al $\pm 1,7 \%$.

Para el diseño del proceder metodológico, se optó por un planteamiento mixto combinando estrategias cuantitativas y cualitativas: entre las primeras, se diseñaron y aplicaron cuestionarios específicos para alumnado, profesorado, equipos directivos y familias; mientras que las estrategias cualitativas estaban orientadas al desarrollo de grupos de discusión con distintos sectores de la comunidad educativa relacionados con la planificación y organización del tiempo escolar. En coherencia con este planteamiento, la búsqueda de explicaciones más amplias acerca de los fenómenos estudiados y de posturas más enriquecidas sobre los problemas de investigación que se pretenden abordar, genera un distanciamiento de la tradicional dicotomía cuantitativo-cualitativo, haciendo emerger con fuerza los métodos mixtos de investigación -Mixed Methods Research (MMR)donde "el investigador mezcla o combina técnicas, métodos, aproximaciones, conceptos y lenguajes cualitativos y cuantitativos en un estudio simple" (ONWEUGBUZIE; JOHNSON, 2004, p. 17).

Para la realización del trabajo de campo se seleccionaron un total de 52 centros teniendo en cuenta las variables antes mencionadas como la titularidad (pública, privada concertada y privada no concertada) y hábitat del centro (rural, urbano). La localización de los centros se hizo al azar, realizándose un 
contacto espontáneo y aleatorio vía telefónica, atendiendo siempre a los criterios de la muestra establecida. Igualmente, la selección de grupos en los cursos de referencia se realizó aleatoriamente. Los cuestionarios fueron aplicados presencialmente en el aula durante el transcurso de la jornada lectiva en cada uno de los centros educativos seleccionados en las 17 Comunidades Autónomas por integrantes del equipo investigador en el curso académico 2010-2011. Posteriormente, se llevó a cabo el análisis estadístico con el paquete SPSS 22.0.

Cuadro 1- Preguntas analizadas del cuestionario de alumnado

\begin{tabular}{|c|c|}
\hline 27. Los días de clase cuánto tiempo dedicas a... & $\begin{array}{l}\text { 31. Los días de clase y en fin de semana cuánto tiempo dedicas a las } \\
\text { siguientes actividades... }\end{array}$ \\
\hline $\begin{array}{l}\text { Jugar con videoconsolas u ordenador } \\
\text { Jugar en casa a otras cosas } \\
\text { Escuchar música } \\
\text { Navegar por internet } \\
\text { Chatear/participar en redes sociales } \\
\text { Leer (libros, revistas, cómics, prensa, etc.) } \\
\text { Estudiar, hacer los deberes } \\
\text { Ayudar en las tareas domésticas } \\
\text { Ayudar en la actividad laboral de tu familia } \\
\text { Estar con la familia } \\
\text { Usar el móvil } \\
\text { Estar sin hacer nada } \\
\text { Ver televisión }\end{array}$ & $\begin{array}{l}\text { Participar en asociaciones, voluntariado, etc } \\
\text { Estar con amigas y amigos fuera del centro escolar } \\
\text { Ir a actividades de música, pintura, etc } \\
\text { Ir a clases particulares } \\
\text { Jugar en la calle, en el parque, etc } \\
\text { Ir de tiendas o de compras } \\
\text { Estar en bares, cafeterías o pubs } \\
\text { Ir al salón de juegos recreativos } \\
\text { Ir a cíber } \\
\text { Salir por la noche } \\
\text { Ir al botellón } \\
\text { Ir a clases de baile, danza, etc } \\
\text { Caminar o pasear } \\
\text { Practicar deporte } \\
\text { Practicar algún hobby }\end{array}$ \\
\hline
\end{tabular}

Fuente: Tiempos escolares y tiempos de ocio: problemáticas específicas y alternativas pedagógico-sociales en la vida cotidiana del alumnado de Educación Secundaria Obligatoria (ESO). Cuestionario aplicado al alumnado en el marco del Proyecto de Investigación PSI2008-04305PSIC. Coordinado por José Antonio Caride et al. (2011).

En este artículo se analizan los resultados de diferentes ítems del cuestionario elaborado y aplicado ad hoc al alumnado adolescente de Educación Secundaria Obligatoria de las diecisiete Comunidades Autónomas. En concreto, pretendíamos analizar la percepción que tiene el alumnado en torno a los usos que realizan de su tiempo libre en los días lectivos y durante los fines de semana; unas preguntas que aparecen recogidas en el cuadro 1.

\section{Resultados}

La actividad predominante en el uso del tiempo libre que realizan los adolescentes en días lectivos es la de compartir momentos familiares a la que dedican más de tres horas diarias, según un 30\% de los alumnos y alumnas que dieron respuesta al cuestionario. Una situación que concuerda con el tiempo que destinan a ayudar en las tareas domésticas ocupando, con distintos niveles de intensidad, entre 15 y 60 minutos de sus actividades diarias $(49,8 \%)$.

En relación al tiempo que dedica el alumnado de ESO a realizar diversas actividades en días lectivos (ver tabla 1), la presencia de las tareas escolares en el tiempo libre tiene una gran incidencia durante la semana, siendo estudiar $y$ hacer los deberes el quehacer al que destinan más tiempo (un 46,5\% indica que invierten de una a tres horas diarias). En la misma línea, cuando se les interroga de forma abierta acerca de cuál es la actividad más importante de todas las que realizan, la respuesta más repetida es la referida a estudiar y realizar tareas académicas.

No obstante, si tenemos en cuenta que un 24,1\% del alumnado encuestado afirma dedicar de una a tres horas a navegar por Internet y un $24,7 \%$ a chatear y utilizar redes sociales, cabe subrayar la notable presencia del ocio digital en la vida cotidiana de los chicos y chicas de esta 
etapa educativa. A ello le sigue el tiempo que dedican a ver la televisión, puesto que un 45,1\% de los encuestados afirma dedicar entre 15 y 60 minutos diarios y un 32,9\% de una a tres horas.

Tabla 1 - Tiempo que dedica el alumnado de ESO a realizar diversas actividades en días lectivos

\begin{tabular}{|c|c|c|c|c|c|}
\hline Actividades/Tiempo dedicado & $\begin{array}{c}\text { Ns-Nc } \\
(\%)\end{array}$ & $\begin{array}{l}\text { Nada-15 } \\
\text { minutos } \\
(\%)\end{array}$ & $\begin{array}{l}\text { De } 15 \text { a } 60 \\
\text { minutos (\%) }\end{array}$ & $\begin{array}{c}\text { De } 1 \text { a } 3 \text { horas } \\
(\%)\end{array}$ & $\begin{array}{c}\text { Más de } 3 \text { horas } \\
(\%)\end{array}$ \\
\hline Jugar con videoconsolas u ordenador & 0,7 & 41,2 & 23 & 18,7 & 6,4 \\
\hline Escuchar música & 1,2 & 37,1 & 38,4 & 15,7 & 7,6 \\
\hline Escuchar la radio & 1,6 & 77,7 & 13,9 & 4,8 & 1,9 \\
\hline Navegar por internet & 2,0 & 27,1 & 38,9 & 24,1 & 7,8 \\
\hline Chatear/Redes sociales & 1,2 & 31,7 & 33,6 & 24,7 & 8,8 \\
\hline Leer & 1,2 & 51,7 & 36,5 & 9,0 & 1,7 \\
\hline Estudiar/Hacer los deberes & 1,5 & 7,5 & 36,0 & 46,5 & 8,6 \\
\hline Ayudar en tareas domésticas & 1,8 & 36,7 & 49,8 & 10,1 & 1,7 \\
\hline Estar con la familia & 1,9 & 11,4 & 30,0 & 26,5 & 30,0 \\
\hline Usar el móvil & 2,2 & 58,1 & 25,2 & 7,4 & 7,0 \\
\hline Estar sin hacer nada & 1,9 & 69,7 & 19,1 & 6,1 & 3,2 \\
\hline Ver televisión & 0,9 & 13,2 & 45,1 & 32,9 & 8,1 \\
\hline
\end{tabular}

Ns-Nc: no sabe / no contesta

Estimación a partir de los ítems que obtienen mayores porcentajes de respuestas en el alumnado, $\mathrm{N}=3306$.

Fuente: Tiempos escolares y tiempos de ocio: problemáticas especificas y alternativas pedagógico-sociales en la vida cotidiana del alumnado de Educación Secundaria Obligatoria (ESO). Cuestionario aplicado al alumnado en el marco del Proyecto de Investigación PSI2008-04305PSIC. Coordinado por José Antonio Caride et al. (2011).

Respecto a las actividades en las que emplean nada o poco tiempo (hasta 15 minutos), se ha de mencionar el escaso hábito que la población de esta franja de edad tiene de escuchar la radio $(77,7 \%)$ o de estar sin hacer nada (69,7\%); una circunstancia que se relaciona, respectivamente, con el gran protagonismo de la imagen frente a otras formas de comunicación y la continua acumulación de tareas por la que se caracterizan los ritmos de vida contemporáneos. En esta franja de edad -de 12 a 16 años- el uso del móvil no es tan acusado como cabría esperar en la juventud, puesto que en días de semana más de la mitad de los encuestados (un 58,1\%) indican que su uso no supera los 15 minutos diarios. Asimismo, un $41,2 \%$ apenas juega con videoconsolas durante los días lectivos, lo que puede explicarse por la existencia de pautas familiares que limitan este tipo de actividades y por la falta de tiempo ante las múltiples responsabilidades académicas y ocupaciones de diverso tipo a las que tienen que dar respuesta durante la semana. 
Tabla 2- Tiempo que dedica el alumnado de ESO a realizar distintas actividades en fines de semana

\begin{tabular}{|c|c|c|c|c|}
\hline Actividades & $\begin{array}{l}\text { Ningún día } \\
(\%)\end{array}$ & $\begin{array}{c}\text { Sábado } \\
(\%)\end{array}$ & $\begin{array}{c}\text { Domingo } \\
(\%)\end{array}$ & $\begin{array}{c}\text { Sábado y Domingo } \\
(\%)\end{array}$ \\
\hline Participar en asociaciones, voluntariado, etc. & 95,1 & 3,0 & 0,8 & 1,1 \\
\hline Estar con amigas/os & 31,6 & 27,6 & 1,4 & 39,4 \\
\hline Jugar en la calle, en el parque, etc. & 72,6 & 9,2 & 2,5 & 15,8 \\
\hline Estar en la calle, en el parque, etc. & 50,0 & 17,9 & 2,5 & 29,5 \\
\hline Ir de tiendas o de compras & 70,3 & 23,0 & 1,5 & 5,3 \\
\hline Salir por la noche & 57,0 & 31,8 & 0,5 & 10,7 \\
\hline Ir al botellón & 78,6 & 17,8 & 0,4 & 3,2 \\
\hline Practicar deporte & 66,0 & 16,0 & 2,8 & 15,3 \\
\hline Practicar algún hobby & 66,2 & 10,5 & 3,9 & 19,4 \\
\hline
\end{tabular}

Estimación a partir de los ítems que obtienen mayores porcentajes de respuestas en el alumnado, N= 3306 .

Fuente: Tiempos escolares y tiempos de ocio: problemáticas especificas y alternativas pedagógico-sociales en la vida cotidiana del alumnado de Educación Secundaria Obligatoria (ESO). Cuestionario aplicado al alumnado en el marco del Proyecto de Investigación PSI2008-04305PSIC. Coordinado por José Antonio Caride et al. (2011).

En la misma línea, tampoco la lectura se presenta como una actividad muy practicada por la población adolescente (un 51,5\% dedican nada o hasta 15 minutos a leer en días lectivos) o si lo hacen, está más vinculada al cumplimiento de tareas académicas -en las que emplean mucho más tiempo- que al gusto o placer que genera la lectura en sí misma.

En cuanto a las actividades que realizan en fin de semana (tabla 2), los datos que se desprenden del cuestionario reflejan la escasa participación de los chicos y chicas en asociaciones $y$ voluntariado, pues un 95,1\% afirman no llevar a cabo actividades de este carácter. En todo caso, el hecho de estar con amigos/as asume un papel protagonista dado que los adolescentes pasan la mayor parte del tiempo de los sábados y domingos $(39,4 \%)$ con su grupo de iguales, reuniéndose en espacios abiertos como la calle o el parque $(29,5 \%)$ y realizando actividades lúdicas (15,8\%).

$\mathrm{Si}$ consideramos exclusivamente en qué emplean el tiempo durante los sábados, destacan dos actividades: salir por la noche $(31,8 \%)$ e ir de compras $(23,0 \%)$. En la etapa de la Educación Secundaria Obligatoria es cuando comienzan las primeras salidas nocturnas de los chicos y chicas, ocupando un papel central en sus fines de semana. También, es el sábado cuando van al botellón -reunirse con amigos para consumir en grupo bebidas alcohólicas de alta graduación- (17,8\%), pese a que cabría esperar resultados de respuesta más elevados. El uso del tiempo libre en actividades vinculadas al consumo, como ir de tiendas, tiene una notable presencia en contraposición a otras prácticas de ocio serio a las que se les atribuyen mayores beneficios para el desarrollo personal y que exigen una mayor dedicación, continuidad y esfuerzo, tal es el caso de hacer deporte $(16,0 \%)$ o practicar algún hobby (10,5\%).

\section{Discusión}

Atendiendo a los resultados obtenidos, aunque en la etapa de la adolescencia el grupo de iguales tiene un papel fundamental en los tiempos de ocio de las chicas y chicos españoles, la familia continúa siendo un pilar esencial. En un estudio realizado con alumnado 
de Educación Secundaria Obligatoria de la provincia de Salamanca (España), Muñoz y Olmos (2010) evidenciaron que los adolescentes se muestran bastante satisfechos con el tiempo que pasan familiarmente, pese a que la relación con sus padres cambia cuantitativamente y los chicos y chicas hablan más con sus amigos, se produce también un cambio cualitativo y siguen necesitando a sus padres a la hora de formar sus valores.

En esta línea, Offer (2013) señala -en una investigación centrada en la asociación existente entre el tiempo familiar y el bienestar emocional de los adolescentes en función del tipo de actividades que realizan juntos- que el ocio familiar es beneficioso para el bienestar de los adolescentes. Por el contrario, el tiempo familiar productivo (hacer los deberes) se asocia con un menor bienestar emocional, al igual que la realización de tareas domésticas. En general, la falta de tiempo familiar refleja un sentimiento prevalente en la sociedad contemporánea referido a que el tiempo para estar juntos ha llegado a ser un gran reto para muchas familias, porque padres e hijos están extremadamente ocupados (BIANCHI et al.; LAREAU; WIGHT et al., 2009 apud OFFER, 2013). Si bien son múltiples las actividades y tareas que realizan los adolescentes fuera del horario lectivo, en nuestro estudio más de la mitad de los chicos y chicas afirman dedicar varias horas al día a estar con su familia, una situación que ha de interpretarse desde el modelo de bienestar familista característico de la sociedad española.

Por otra parte, ha de subrayarse la notable presencia del ocio digital centrado fundamentalmente en el uso de Internet y de las redes sociales, que han adquirido una presencia cada vez mayor en los procesos de socialización de los adolescentes y jóvenes, llegando a convertirse en su principal agente de socialización (PALLARÉS, 2014). A este respecto, para Sádaba y Bringué (2010) pueden realizarse diversas lecturas acerca de la ocupación digital del tiempo por parte de los adolescentes: de un lado, supone el uso de medios diversos para entretenerse, socializarse, estar en contacto con su grupo de iguales o desarrollar su identidad a través de la interactividad que ofrecen dichos medios; de otro, es un motivo de preocupación para las familias por la repercusión negativa que puede tener la realización de un uso inadecuado de las tecnologías: acceso a contenidos inapropiados, exposición de la privacidad e intimidad de los menores a ojos de extraños, etc. A ello, se ha de añadir que, en general, los padres aprenden acerca de los medios digitales después que sus hijos y ven limitado el impacto de las actuaciones educativas que ellos pueden llegar a ejercer en este ámbito.

Otro aspecto de interés en el estudio del ocio y de los tiempos libres de los adolescentes desde una perspectiva educativa, tiene que ver con sus prácticas de lectura. Los datos presentados evidencian la necesidad de promover hábitos lectores entre los chicos y chicas de Secundaria Obligatoria, debido a que la lectura constituye una actividad poco frecuente en sus experiencias de ocio a pesar de su relevancia en la interpretación del mundo que les rodea y en el desarrollo de una visión crítica de la realidad. No obstante, según el barómetro de Hábitos de lectura y compra de libros en España (ESPAÑA, 2012), el mayor porcentaje de lectores en el contexto español se sitúa -por edades- en el rango de edad de 14 a 24 años; un porcentaje que va decreciendo a medida que aumenta la edad.

Además, debe tenerse en cuenta la tendencia alcista en el número de lectores en soporte digital, pues en el año 2012 -según el mismo barómetro- nueve de cada diez jóvenes de 14 a 34 años, leía en soportes digitales. En esta línea, Gil Flores (2011) en una investigación llevada a cabo en el tercer curso de Educación Secundaria Obligatoria en centros públicos y privado-concertados de la Comunidad Autónoma de Andalucía (España), indicaba que la lectura en Internet es la forma de lectura más frecuente entre los alumnos y alumnas de dicho curso; afirmando también que en los tiempos de ocio la práctica de la lectura no sólo contribuye 
al disfrute y al desarrollo personal sino además a la potenciación de otras habilidades esenciales para un aprendizaje a lo largo de toda la vida.

La escuela y otros agentes e instituciones educativas (bibliotecas, centros culturales, centros de ocio, etc.) -en convergencia con las familias- han de asumir el reto de fomentar el gusto por la lectura entre los chicos y chicas, combinando los formatos tradicionales con nuevos soportes técnicos que favorezcan la aproximación a este tipo de actividades. Desde esta perspectiva, coincidimos con Muñoz y Hernández (2011, p. 623) en que la educación en el tiempo libre "resulta fundamental como paso previo al fomento de la lectura, al menos más allá del entorno escolar”, no sólo para que los adolescentes accedan al conocimiento sino también para que se construyan como personas y desarrollen su propia identidad.

A tenor de los datos obtenidos, la participación de los y las jóvenes en tareas vinculadas a la acción social y el voluntariado resulta prácticamente inexistente. Unos resultados que evidencian la necesidad de orientar los procesos educativos y los esfuerzos de diversos agentes comunitarios hacia prácticas que estimulen un mayor sentimiento de identidad, integración y responsabilidad, entendiendo que

[...] construir comunidad exige interesarse por lo que sucede en el entorno próximo, y participar en las diversas dinámicas sociales orientadas a crear oportunidades para la práctica de la solidaridad colectiva y el ejercicio de la responsabilidad individual en función del bien común (MORÁN et al., 2012, p. 95).

Por tanto, nos enfrentamos a un doble reto para la Pedagogía del Ocio: de un lado, se trata de potenciar experiencias e iniciativas que promuevan la participación de los adolescentes en iniciativas comunitarias; $y$, de otro, concienciar a los jóvenes acerca de la necesidad de asumir un compromiso sólido con la transformación de las situaciones de injustica y la mejora de los entornos en los que viven.

Por último, se evidencian diferencias respecto de las prácticas de ocio de los adolescentes durante la semana y en los fines de semana; así, gran parte de las actividades que realizan durante el fin de semana tienen lugar los sábados, predominando el salir por la noche, estar con los amigos e ir de compras. En este sentido, coincidimos con Ortega y Madariaga (2013), en que uno de los factores que más influye en las preferencias de las personas para realizar celebraciones es el hecho de estar con los amigos; de ahí que la gente tenga especial predilección por tener experiencias extraordinarias que posibilitan interactuar con los demás. Específicamente, para los jóvenes españoles y según datos del INJUVE (ESPAÑA, 2013), el ocio nocturno se asocia con el hecho de romper con la rutina, disponer de un momento para ellos mismos, etc.; si bien, minoritariamente también se vincula con ideas de liberación, encanto, sensación de libertad y de no control.

En concordancia con estas afirmaciones, las actividades que realizan en el tiempo de ocio nocturno se centran en ir de bares, cafeterías, salir a bailar, acudir a casa de algún amigo o ir al cine. No obstante, en los segmentos más jóvenes -es decir, en los menores de 24 añosdestaca el ir de botellón; una actividad donde el consumo de alcohol con el grupo de iguales en espacios públicos (parques, plazas, etc.) cobra mayor protagonismo. Así, para Comas (2000), el consumo de alcohol fuera de los lugares pautados sigue los mismos patrones de horario y consumo que el ocio comercial; sin embargo, no constituye una forma de provocación, sino un comportamiento perfectamente integrado que puede estar relacionado con un potencial rechazo hacia un modelo de ocio económicamente inaccesible para muchos jóvenes.

En este marco, merece atención el tiempo que los adolescentes dedican los fines de semana a ir de compras; pues según Osuna (2008), los adolescentes manifiestan cierta 
preferencia por ir de compras con sus amigos, aunque también les gusta ir acompañados por familiares, fundamentalmente de sus madres. No obstante, los jóvenes de entre 12 y 18 años, quieren parecerse a otros grupos de edad y están desarrollando hábitos de consumo propios de los adultos (BLASCO; PINTAD0, 2014).

\section{Conclusiones}

Los hallazgos presentados nos llevan a subrayar algunas apuestas estratégicas que atañen al campo pedagógico-social en la tarea de educar el ocio adolescente, y que se sitúan en visibilizar los valores y significados que aporta el ocio al desarrollo humano, como una tarea en la que la pedagogía debe comprometerse activamente (CARIDE, 2012). En coherencia con esta afirmación, la educación en un ocio liberador, ha de potenciar la autonomía de los jóvenes así como posibilitar que aprendan a ser agentes activos de sus itinerarios de ocio, considerando factores como el sentirse libres para tomar decisiones autónomas y propias, involucrase con las decisiones tomadas y poder expresar sus deseos (FRANCILEUD0; DE OLIVEIRA; NIEVA, 2014).

El tránsito de un ocio casual a un ocio serio o valioso, exige una apuesta decidida por la educación de los adolescentes en su tiempo libre así como la asunción de responsabilidades compartidas por parte de diferentes agentes educativos que tienen una incidencia clave en el desarrollo integral de los chicos y chicas. Si bien, el ocio casual requiere unas mínimas habilidades y conocimiento, el ocio serio -esto es, la práctica sistemática de una actividad amateur, de voluntariado o un hobby- exige esfuerzo, perseverancia y -en general- un fuerte sentido de pertenencia a un determinado grupo social (STEBBINS, 2012). Además, la participación constituye un elemento esencial en sus itinerarios lúdicos, recreativos y de ocio ya que

[...] cuanto más comprometidos estén en los procesos de diseño y cuidado de los espacios para el ocio compartido con sus iguales, mayores serán los retornos socioeducativos que los jóvenes extraerán de sus experiencias de ocio. (ORTEGA; BAYÓN, 2014, p. 11).

En definitiva, tal y como recoge, en su artículo 2.4, la Carta Internacional de la Educación del Ocio ${ }^{1}$ (WLRA, 1994), el ocio es un derecho humano básico y fundamental, y su desarrollo requiere de la articulación de una acción coordinada entre los gobiernos, las asociaciones no gubernamentales, el voluntariado, las instituciones educativas $\mathrm{y}$ los medios de comunicación, permitiendo que los adolescentes y jóvenes logren alcanzar su máximo potencial en ocio. En consecuencia, será una responsabilidad compartida de todos los agentes que viabilizan las prácticas educativas, generar y desarrollar alternativas de ocupación y disfrute del tiempo libre que sean enriquecedoras para los adolescentes y jóvenes; pero no con la intención de limitar su protagonismo, sino con el firme propósito de intensificarlo y acompañarlos educativamente en la construcción de sus experiencias y vivencias del ocio.

1- International Charter for Leisure Educaction. 


\section{Referencias}

BLASCO, Francis; PINTADO, Teresa. Adolescentes 2013: hacia una correcta formación y autonomía en el consumo. El Ejido: Círculo Rojo, 2014.

CABALLO, María Belén; CARIDE, José Antonio; MEIRA, Pablo. El tiempo como contexto y pretexto educativo en la sociedad red. Educación Social, Barcelona, n. 27, p. 11-27, 2011.

CARIDE, José Antonio. Lo que el tiempo educa: el ocio como construcción pedagógica y social. Arbor, Madrid, v. 188, n. 754, p. 301-313, 2012.

COMAS, Domingo. Agobio y normalidad: una mirada crítica sobre el sector del ocio juvenil en la España actual. Revista de Estudios de Juventud, Madrid, n. 50, p. 9-22, 2000.

COMAS, Domingo. Las políticas de juventud en la España democrática. Madrid: Injuve, 2007.

CUENCA, Manuel. Ocio y formación: hacia la equiparación de oportunidades mediante la educación de ocio. Bilbao: Universidad de Deusto, 1999.

DUMAZEDIER, Joffre. Hacia una civilización del ocio. Barcelona: Estela, 1964.

ECHEBURÚA, Enrique; LABRADOR, Francisco Javier; BECOÑA, Elisardo (Coord.). Adicción a las nuevas tecnologías en adolescentes y jóvenes. Madrid: Pirámide, 2009.

ESPAÑA. Federación de Gremios de Editores de España. Hábitos de lectura y compra de libros en España 2012. Toledo: Ministerio de Educación, Cultura y Deporte, 2013. Disponible en: <http://www.editoresmadrid.org/media/43692/h\%C3\%A1bitos\%20 lectura\%20a\%C3\%B10\%202012.pdf>. Acceso en: 30 jun. 2015.

ESPAÑA. Informe de la juventud en España 2012. Madrid: Ministerio de Sanidad, Servicios Sociales e lgualdad: INJUVE, 2013. Disponible en: <http://www.injuve.es/sites/default/files/2013/26/publicaciones/JJE2012_0.pdf>. Acceso: 16 jun. 2015.

FRANCILEUDO, Francisco Antonio; DE OLIVEIRA, José Clerton; NIEVA, Fabiana. La relación entre la pedagogía del ocio y prácticas educativas en familias con hijos adolescentes. In: ORTEGA, Cristina; BAYÓN, Fernando (Coord.). El papel del ocio en la construcción social del joven. Bilbao: Universidad de Deusto, 2014. p. 211-226.

GIL FLORES, Javier. Hábitos lectores y competencias básicas en el alumnado de Educación Secundaria Obligatoria. Educación XX1, Madrid, n. 14, p. 117-134, 2011.

GRADAílLE, Rita; VARELA, Laura; DE VALENZUELA, Ángela. Preocupaciones del profesorado y de las familias sobre los tiempos escolares y de ocio del alumnado de educación secundaria postobligatoria. Revista Interuniversitaria de Formación del Profesorado, Zaragoza, n. 86 (30.2), p. 49-62, 2016.

LASÉN, Amparo. A contratiempo: un estudio de las temporalidades juveniles. Madrid: Centro de Investigaciones Sociológicas CIS, 2000.

MERINO, José Vicente. Jóvenes y educación para el ocio: el reto de las estrategias. In: BELTRÁN, Jesús et al. (Coord.). Familia, juventud y nuestros mayores: la actitud proactiva. A Coruña: Fundación Caixa Galicia, 2001. p. 169-216.

MONTEAGUDO, María Jesús. Reconstruyendo la experiencia de ocio: características, condiciones de posibilidad y amenazas de la sociedad de consumo. In: MONTEAGUDO, María Jesús (Ed.). La experiencia de ocio: una mirada científica desde los Estudios de Ocio. Bilbao: Universidad de Deusto, 2008. p. 81-110.

MORÁN, Carmen et al. Usos e imágenes del tiempo en el alumnado de Educación Secundaria Obligatoria (ESO): entre la escuela, la familia y la comunidad. Pedagogía Social. Revista interuniversitaria, Salamanca, n. 20, p. 61-101, 2012. 
MUÑOZ, José Manuel; HERNÁNDEZ, Azucena. Hábitos lectores de los alumnos de la ESO en la provincia de Salamanca. ¿Son el género y el entorno factores diferenciales? Revista de Educación, Madrid, n. 354, p. 605-628, 2011.

MUÑOZ, José Manuel; OLMOS, Susana. Adolescencia, tiempo libre y educación: un estudio con alumnos de la ESO. Educación XX1, Madrid, v. 13, n. 2, p. 139-162, 2010.

NACIONES UNIDAS. Asamblea General. Programa de Acción Mundial para los Jóvenes hasta el año 2000 y años subsiguientes. Resolución aprobada por la Asamblea General [sin remisión previa a una Comisión Principal (A/50/728)] 50/81, 1996. Disponible en: <http://www.cinu.mx/minisitio/UNjuventud/docs/Programa_accion_mundial_jovenes.pdf> Acceso en: 30 jun. 2015.

OFFER, Shira. Family time activities and adolescents' emotional well-being. Journal of Marriage and Family, Austin, v. 75, n. 1, p. 26-41, 2013.

ONWUEGBUZIE, Anthony; JOHNSON, Burke. Mixed methods research: a research paradigm whose time has come. Educational Researcher, Florida, v. 33, n. 7, p. 14-26, 2004.

ORTEGA, Cristina; BAYÓN, Fernando (Coord.). El papel del ocio en la construcción social del joven. Bilbao: Universidad de Deusto, 2014.

ORTEGA, Cristina; MADARIAGA, Aurora. Festive leisure: significant changes in perception and organization of celebrations. Journal of Tourism, Research \& Hospitality, Los Ángeles, v. 2, n. 3, p. 1-8, 2013.

OSUNA, Sara. Publicidad y consumo en la adolescencia. Barcelona: Icaria, 2008.

PALLARÉS, Marc. Medios de comunicación: ¿espacio para el ocio o agentes de socialización en la adolescencia? Pedagogía Social. Revista interuniversitaria, Salamanca, n. 23, p. 231-252, 2014.

PUIG, Josep María; TRILLA, Jaume. La pedagogía del ocio. Barcelona: Laertes, 1996.

SÁDABA, Charo; BRINGUÉ, Xavier. Niños y adolescentes españoles ante las pantallas: rasgos configuradores de una generación interactiva. CEE Participación Educativa, Madrid, n. 15, p. 86-104, 2010. Disponible en: <http://www.mecd.gob.es/revista-cee/ pdf/n15-bringue-sala.pdf>. Acceso en: 17 jun. 2015.

SALVADOR, Teresa. Adolescentes y jóvenes: ocio y usos del tiempo libre en España. Madrid: Delegación del Gobierno para el Plan Bacional de Drogas, 2009. Disponible en: <http://www.pnsd.msc.es/novedades/pdf/RevisionAdolescentes.pdf>. Acceso en: 30 jun. 2015.

STEBBINS, Robert. Compromiso temporal discrecional: efectos sobre la elección y el estilo de ocio. Arbor, Madrid, v. 188, p. 293-300, 2012.

TRILLA, Jaume. Perspectivas educativas del ocio para el siglo XXI. Proyecto Hombre, Madrid, n. 32, p. 8-13, 1999.

WLRA - World Leisure and Recreation Association. International Charter for Leisure Education - ELRA, 1994. In: CUENCA, Manuel. Pedagogía del ocio: modelos y propuestas. Bilbao: Universidad de Deusto, 2004. p. 315-324.

Recibido en: 23.07.2015

Aprobado en: 09.08.2016

Laura Varela Crespo es doctora en ciencias de la educación con premio extraordinario de doctorado. Diplomada en educación social y licenciada en pedagogía. Profesora en el Departamento de Pedagogía y Didáctica de la Universidad de Santiago de Compostela y miembro del grupo de investigación SEPA-interea, reconocido como grupo de referencia competitiva por la Xunta de Galicia.

Rita Gradaílle Pernas es doctora en ciencias de la educación con premio extraordinario de doctorado. Diplomada en magisterio y licenciada en pedagogía, también con premio extraordinario. Profesora en el Departamento de Pedagogía y 
Didáctica de la Universidad de Santiago de Compostela y miembro del grupo de investigación SEPA-interea, reconocido como grupo de referencia competitiva por la Xunta de Galicia.

Yésica Teijeiro Bóo es licenciada en pedagogía. Profesora en el Departamento de Pedagogía y Didáctica de la Universidad de Santiago de Compostela y miembro del grupo de investigación SEPA-interea, reconocido como grupo de referencia competitiva por la Xunta de Galicia. 